\title{
Friabilidad del suelo: métodos de estimación con énfasis en la determinación cuantitativa de la resistencia al rompimiento
}

\author{
Soil friability: estimation methods with emphasis on \\ the quantitative determination of breaking resistance
}

\author{
Friabilidade do solo: métodos de estimação com um \\ ênfase na determinação quantitativa da força de ruptura
}

\section{Edgar Álvaro Ávila Pedraza}

Agrólogo, Magíster en Ciencias Agrarias Línea de Investigación en Suelos y Aguas, Doctor en Ciencias Agrarias Línea de Investigación Suelos y Aguas.

Departamento de Suelos y Aguas. Facultad de Ingeniería Agronómica. Universidad del Tolima. Ibagué. Colombia

eaavila@ut.edu.co

\section{Resumen}

Entender el comportamiento de la friabilidad permite la toma de decisiones apropiadas para la reducción del consumo de energía, el manejo y la conservación de la calidad física del suelo. Este artículo sintetiza los métodos utilizados actualmente para la determinación de la friabilidad del suelo, con énfasis en el método de resistencia al rompimiento -RR-. La friabilidad puede ser determinada a partir de métodos cualitativos, semicuantitativos y cuantitativos. El análisis cualitativo se basa en el examen visual de la estructura y consistencia del suelo, mientras que el análisis semi-cuantitativo se centra en la cuantificación de la distribución de agregados o fragmentos producto del sometimiento del suelo a impacto por caída. Los métodos cuantitativos permiten determinar la friabilidad de forma directa mediante la resistencia del suelo, o de forma indirecta, por inferencia a partir del comportamiento de otras propiedades del suelo: curva de retención de agua, porosidad y funciones de pedotransferencia. El método cuantitativo de RR se realiza mediante la compresión de agregados naturales o remoldeados de suelo hasta lograr su fractura, y es uno de los más aceptados. Los métodos cualitativos se realizan directamente en campo, de manera fácil y rápida pero su resultado es menos confiable. Los métodos semicuantitativos, rompimiento del suelo por caída, pueden realizarse en campo o laboratorio pero no ofrecen información precisa sobre la resistencia del suelo. Los métodos cuantitativos, implican el transporte de las muestras al laboratorio, mayor tiempo de ejecución y equipos y personal especializado, pero sus resultados son más confiables y reproducibles.

Palabras clave: índice de friabilidad, agregados del suelo, calidad del suelo, resistencia a la compresión, labranza del suelo.

\section{Abstract}

Understand the behavior of the friability allows the taking of appropriate decisions for the reduction of energy consumption, the management and conservation of the physical quality of the soil. This article summarizes the methods currently used for the 
determination of the friability of soil, with emphasis on the method of resistance to tearing - RR-. The friability can be determined on the basis of qualitative methods, semiquantitative and quantitative. The qualitative analysis is based on visual examination of the structure and consistency of the soil, while the analysis semi-quantitative focuses on the quantification of the distribution of aggregates or fragments product of the subjection of the ground to impact by fall. Quantitative methods to determine the friability directly through the soil resistance, or indirectly, by inference from the behavior of other soil properties: curve of water retention, porosity and pedotransfer functions. The quantitative method of RR is performed using the compression of natural aggregates or remoldeados of soil to achieve its fracture, and is one of the most accepted. Qualitative methods are performed directly in the field, in an easy and fast way but its result is less reliable. The methods semiquantitative, breaking of the soil by fall, can be carried out in the field or laboratory but do not provide accurate information on the resistance of the soil. Quantitative Methods, involve the transport of samples to the laboratory, greater execution time and equipment and specialized personnel, but their results are more reliable and reproducible.

Key-words: friability index, soil aggregates, soil quality, compressive strength, soil tillage.

\section{Resumo}

Entender o comportamento da friabilidade permite tomar decisões apropriadas para a redução do consumo de energia, manejo e conservação da qualidade física do solo. Este artigo sintetiza os métodos utilizados atualmente para a determinação da friabilidade do solo, focando no método de força de ruptura -RR-. A friabilidade pode ser determinada a partir de métodos qualitativos, semiquantitativos e quantitativos. A análise qualitativa baseisa-se no exame visual da estrutura e consistência do solo, enquanto a análise semi-quantitativa foca-se na quantificação da distribuição de agregados ou fragmentos, produto do sometimento do solo ao impacto por queda. Os métodos quantitativos permitem determinar a friabilidade de forma direta por médio da resitencia do solo, ou de forma indireta, inferindo a partir do comportamento de outras propriedades do solo: curva de retenção de água, porosidade e funções de pedotransferência. O método quantitativo de RR realiza-se por compressão de agregados naturais ou remoldados do solo até conseguir seu quebramento, sendo um dos mais aceitos. Os métodos qualitativos realizam-se diretamente no campo, de forma fácil e rápida mas o resultado não é tão confiável. Nos métodos semiquantitativos, o quebramento do solo por queda, pode ser feito no campo ou laboratório mas, não oferecem imformação precisa sobre a resistência do solo. Os métodos quantitativos involucram o transporte de amostras de laboratório, maior tempo de execução e equipos e pessoal especializado, mas seus resultados são mais confiáveis e reproducíveis.

Palavras-chave: índice de friabilidade, agregados do solo, qualidade do solo, resistência à compressão, lavoura do solo.

\section{Introducción}

La friabilidad es una propiedad física esencial para la determinación de la respuesta del suelo a las operaciones de labranza y un factor clave en la obtención de una capa arable con el tamaño de agregados adecuado para el crecimiento de las raíces (Munkholm, 2011). En estado friable los suelos requieren una labranza mínima para producir una adecuada cama de siembra y, por el contrario, aquellos que no lo están, pueden requerir alta demanda de energía en la labranza y el resultado final es un lecho de siembra inadecuado para la germinación, emergencia y establecimiento de plantas (Mullins et al., 1990; Macks et al., 1996). Adicionalmente, el suelo en estado friable presenta menor riesgo a la degradación estructural (Macks et al., 1996). 
Utomo \& Dexter (1981) y Dexter \& Watts (2001) definen la friabilidad como la tendencia de una masa de suelo no confinada a desmoronarse en un intervalo particular de agregados de menor tamaño bajo un esfuerzo aplicado. Lo que se desea con el estado friable del suelo es que los agregados más grandes -terrones-, al romperse por efecto de un esfuerzo aplicado, generalmente el uso de implementos de labranza, se fragmenten en un conjunto homogéneo de agregados más pequeños resistentes a la degradación y cuyo tamaño ofrezca un medio óptimo para el desarrollo del sistema radical de las plantas. Una buena cama de siembra es uno de los objetivos de la labranza, actividad que se lleva a cabo más fácilmente en suelos friables en que los terrones grandes son relativamente débiles y los agregados pequeños son relativamente fuertes y resistentes al desmoronamiento (Dexter, 1997; Schjǿnning et al., 2002). Watts \& Dexter (1998), consideran la friabilidad como un indicador de la calidad estructural del suelo y Macks et al. (1996), la sugieren como un indicador de la condición estructural para la plantación de cultivos en siembra directa. De acuerdo con Karlen et al. (1990), un suelo en estado friable es una condición asociada a una adecuada labranza.

Para la estimación de la friabilidad se han empleado métodos cualitativos, semi-cuantitativos y cuantitativos (Munkholm, 2011). Los métodos cualitativos han sido ampliamente utilizados y se basan en la descripción de la morfología y grado de desarrollo de la estructura y la determinación de la consistencia del suelo en húmedo (USDA, 2002; FAO, 2009 y Ball et al., 2007). Los métodos semi-cuantitativos aplican el impacto de caída para fragmentar el material de suelo y establecer características como la distribución del tamaño de agregados, su diámetro geométrico medio o el diámetro promedio ponderado (Hadas \& Wolf, 1984). Los métodos cuantitativos en su mayoría se basan en la determinación de la resistencia de los agregados del suelo, bien sea naturales o remoldeados. Se han documentado otros métodos cuantitativos para determinar la friabilidad en forma indirecta: i) relación de la friabilidad con la curva de retención de agua (Dexter, 2004a, Dexter, 2004b y Dexter \& Bird, 2001); ii) relación de la friabilidad con la porosidad estructural (Guérif, 1990 y Munkholm et al., 2002b) y iii) a partir de funciones de pedotransferencia. Algunos autores como Snyder et al. (1995), consideran que es necesario establecer ensayos de friabilidad rápidos y sencillos que involucren pequeñas muestras de suelo. Uno de los métodos cuantitativos más aceptados es el de la RR (Utomo \& Dexter, 1981; Watts \& Dexter, 1998 y Dexter \& Watts, 2001), produciendo la ruptura de agregados de suelos mediante un esfuerzo aplicado -compresión-, en condiciones de laboratorio, y, a través de la determinación del coeficiente de variación de la $R R$, expresado a través de un intervalo de confianza, se determina un índice de friabilidad.

El objetivo de este artícuulo es presentar una síntesis de los principales métodos cualitativos, semicuantitativos y cuantitativos para la determinación de la friabilidad del suelo, con especial énfasis en el método de resistencia al rompimiento.

\section{Métodos para determinar la friabilidad del suelo}

De acuerdo con Munkholm (2011), la friabilidad ha sido evaluada mediante la aplicación de métodos cualitativos de campo, semicuantitativos de campo y laboratorio, y cuantitativos realizados en condiciones controladas de laboratorio. En la Figura 1 se presentan los principales métodos utilizados en la evaluación de la friabilidad del suelo. 


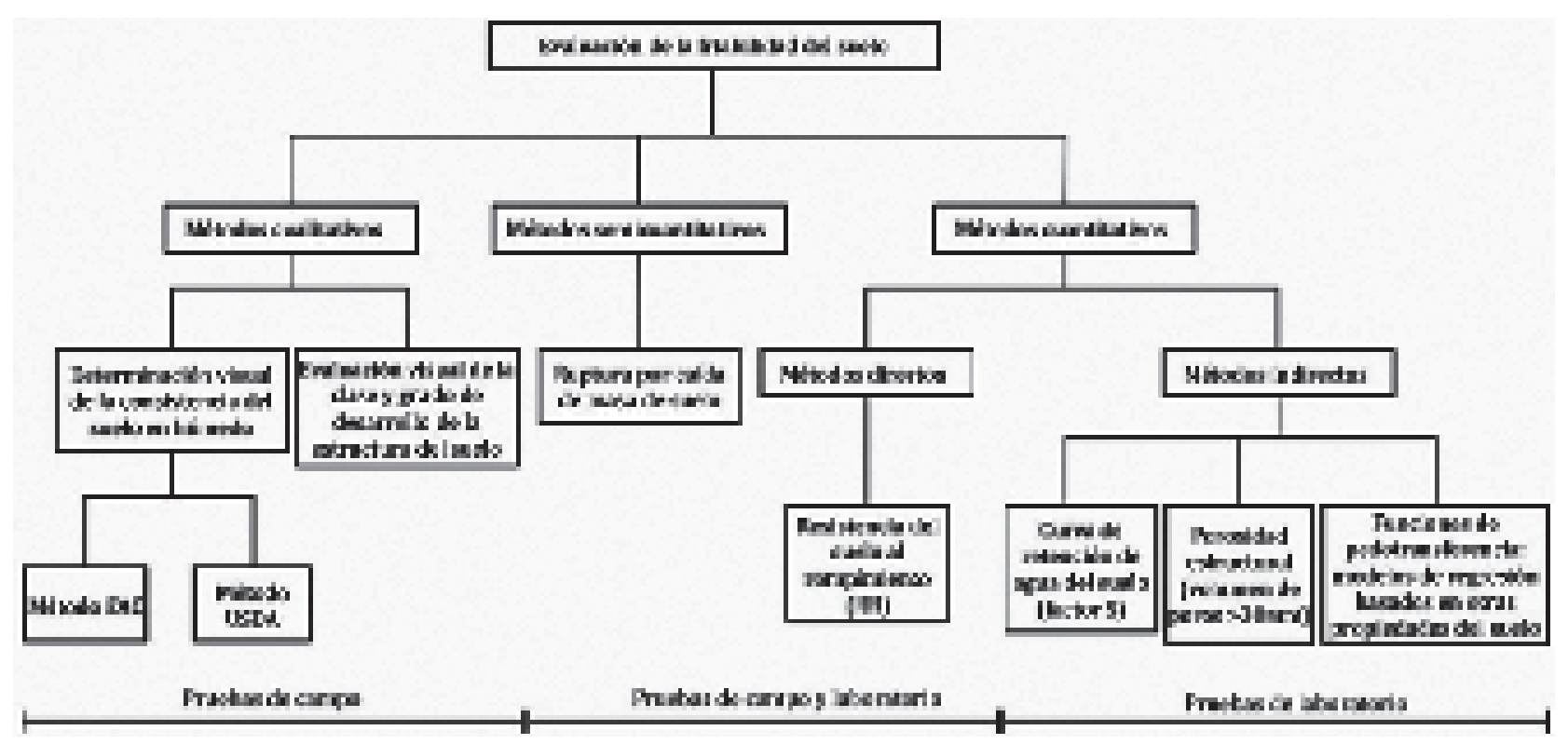

Figura 1. Principales métodos utilizados para evaluar la friabilidad del suelo.

\section{Métodos cualitativos}

Se conocen principalmente dos métodos de evaluación cualitativa: a partir de la determinación de la consistencia del suelo en húmedo (USDA, 2002 y FAO, 2009), y a través de la evaluación de su estructura (Ball et al., 2007).

\subsection{Determinación visual de la consistencia del suelo en húmedo}

Esta determinación se realiza normalmente cuando se lleva a cabo la descripción de la consistencia en los perfiles modales de suelos en los levantamientos edafológicos y se utilizan fundamentalmente dos métodos:

Método USDA: de acuerdo con USDA (1993), la consistencia se refiere a los atributos del material de suelo que se expresan con relación al grado de adhesión y cohesión o la resistencia a la deformación o ruptura. La consistencia es altamente dependiente de la condición de humedad y su descripción no tiene mucho sentido si no se especifica conjuntamente el contenido de agua del suelo. Para determinar la friabilidad por este método se coloca un agregado o terrón del suelo en estado húmedo entre el índice y el pulgar (Figura 2) o debajo del pie, y se aplica un esfuerzo de compresión que permita desmoronarlo o romperlo; en el caso de suelos muy consistentes que no se puedan fragmentar con la mano o el pie, el material de suelo se golpea contra una superficie dura. Para determinar la friabilidad por este método, se utilizan los criterios que aparecen en la Tabla 1.

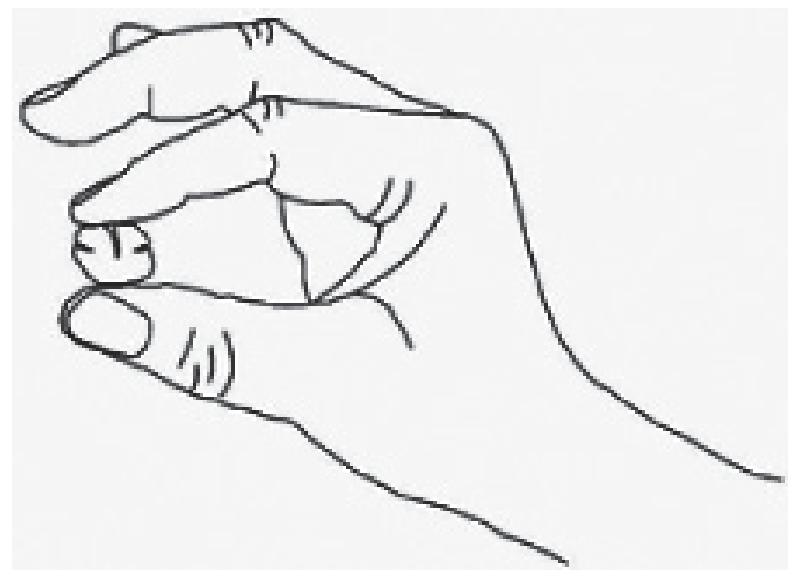

Figura 2. Determinación de la friabilidad del suelo de manera manual. 
Tabla 1. Categorías consideradas en la clasificación de la consistencia del suelo en húmedo

\begin{tabular}{|c|c|c|c|}
\hline \multicolumn{2}{|c|}{ Consistencia en húmedo } & \multicolumn{2}{|c|}{ Descripción de la prueba } \\
\hline Clase & Código & Operación & Fuerza aplicada \\
\hline Suelta & $S$ & No se puede obtener fragmento intacto & \\
\hline Muy friable & MFR & Fuerza muy pequeña entre los dedos & $<8 \mathrm{~N}$ \\
\hline Friable & $\mathrm{FR}$ & Ligera fuerza entre los dedos & $8 a<20 N$ \\
\hline Firme & $\mathrm{FI}$ & Moderada fuerza entre los dedos & $20 \mathrm{a}<40 \mathrm{~N}$ \\
\hline Muy firme & MFI & Gran fuerza entre los dedos & $40 \mathrm{a}<80 \mathrm{~N}$ \\
\hline Extremadamente firme & EFI & Fuerza moderada entre las manos & $80 a<160 N$ \\
\hline Ligeramente rígida & LR & Presión del pie con el peso del cuerpo & $160 a<800 N$ \\
\hline Rígida & $\mathrm{R}$ & Golpe leve & $800 \mathrm{~N} \mathrm{a}<3 \mathrm{~J}$ \\
\hline Muy rígida & MR & Golpe moderado a fuerte & $\geq$ a $3 \mathrm{~J}$ \\
\hline
\end{tabular}

$\mathrm{N}=$ Newton; J= Julio Fuente: USDA, 2002.

Método FAO: la consistencia se refiere al grado de cohesión o adhesión de la masa del suelo e incluye propiedades de este relacionadas entre sí como la friabilidad, plasticidad, adhesividad y resistencia a la compresión (FAO, 2009). Este método de determinación de la friabilidad guarda gran similitud con el establecido por USDA (2002), y consiste en describir la consistencia del suelo en las condiciones naturales de humedad del perfil; cuando se utiliza esta técnica en perfiles de suelo seco, se sugiere humedecerlo hasta llevarlo a una medida subjetiva próxima a capacidad de campo. En este método la consistencia se determina mediante el rompimiento de una masa de suelo entre los dedos y su clasificación se realiza con base en la respuesta del material de suelo al comprimirlo, en las categorías que se observan en la Tabla 2.

Tabla 2. Clasificación de la consistencia del suelo en húmedo

\begin{tabular}{|c|c|c|}
\hline Código & $\begin{array}{l}\text { Tipo de consistencia } \\
\text { en húmedo }\end{array}$ & Características del material de suelo \\
\hline LO & Suelto & No coherente \\
\hline VFR & Muy friable & $\begin{array}{l}\text { El material de suelo se aplasta bajo presión leve entre los dedos, } \\
\text { pero es coherente cuando se lo presiona todo al mismo tiempo }\end{array}$ \\
\hline FR & Friable & $\begin{array}{l}\text { El material de suelo se aplasta fácilmente bajo presión suave a moderada } \\
\text { entre los dedos, y se vuelve coherente cuando se lo presiona junto }\end{array}$ \\
\hline $\mathrm{FI}$ & Firme & $\begin{array}{l}\text { El material de suelo se aplasta bajo presiones moderadas entre } \\
\text { los dedos, pero su resistencia es mayor que la anterior categoría }\end{array}$ \\
\hline VFI & Muy firme & $\begin{array}{l}\text { El material de suelo se aplasta a presión fuerte entre los dedos; } \\
\text { difícilmente aplastable entre los dedos }\end{array}$ \\
\hline EFI & Extremadamente firme & $\begin{array}{l}\text { El material de suelo se aplasta solo a presiones muy fuertes; } \\
\text { no puede aplastarse entre los dedos }\end{array}$ \\
\hline
\end{tabular}




\subsection{Evaluación visual de la clase y grado de desarrollo de la estructura del suelo}

Este método se basa en una modificación del inicialmente propuesto por Peerlkamp (1959), quien desarrolló una escala para evaluar la calidad de la estructura del suelo. Es un método de determinación de la friabilidad que generalmente se realiza directamente en campo y que comprende fundamentalmente tres etapas: extracción y revisión del bloque de suelo, ruptura del bloque y asignación de la puntuación al suelo con base en la descripción de la estructura (Ball et al., 2007). A partir de los bloques que se extraen de cada horizonte, normalmente los superficiales, con la ayuda de una pala, se separan los agregados por sus planos de debilidad natural y se dejan expuestos para su observación los diferentes tamaños de agregados. En los agregados obtenidos se evalúa, mediante la utilización de una tabla guía, características como tamaño, forma, presencia de aristas y porosidad. Como este método se trabaja de manera independiente para cada horizonte del suelo, la evaluación final corresponde al producto de la puntuación de cada horizonte por su espesor y se pondera por la profundidad total considerada. En la Tabla 3 se presentan las categorías de evaluación consideradas en este método.

Tabla 3. Clasificación de la friabilidad del suelo basada en la evaluación visual de la estructura

\begin{tabular}{|c|c|c|c|c|c|}
\hline Código & $\begin{array}{l}\text { Calidad } \\
\text { de la } \\
\text { estructura }\end{array}$ & $\begin{array}{c}\text { Facilidad } \\
\text { de ruptura } \\
\text { (suelo húmedo) }\end{array}$ & $\begin{array}{c}\text { Tamaño y apariencia } \\
\text { de los agregados }\end{array}$ & Porosidad visible & Raíces \\
\hline Sq1 & $\begin{array}{l}\text { Friable (se des- } \\
\text { morona el bloque } \\
\text { y tiende a caer de } \\
\text { la pala). }\end{array}$ & $\begin{array}{l}\text { Los agregados } \\
\text { se desmoronan } \\
\text { fácilmente con los } \\
\text { dedos. }\end{array}$ & $\begin{array}{l}<\text { de } 6 \mathrm{~mm} \text { después } \\
\text { de desmoronarse. }\end{array}$ & $\begin{array}{l}\text { Altamente } \\
\text { porosos. }\end{array}$ & $\begin{array}{l}\text { Raíces en todo } \\
\text { el suelo. }\end{array}$ \\
\hline Sq2 & $\begin{array}{l}\text { Intacta (el bloque } \\
\text { se mantiene con } \\
\text { su forma original } \\
\text { en la pala). }\end{array}$ & $\begin{array}{l}\text { Los agregados se } \\
\text { rompen fácilmente } \\
\text { con la mano. }\end{array}$ & $\begin{array}{l}\text { Una mezcla de agregados } \\
\text { porosos, redondeados, de } \\
2 \text { a } 70 \mathrm{~mm} \text {. No se presen- } \\
\text { tan terrones. }\end{array}$ & $\begin{array}{l}\text { La mayoría de los } \\
\text { agregados son } \\
\text { porosos. }\end{array}$ & $\begin{array}{l}\text { Raíces en todo } \\
\text { el suelo. }\end{array}$ \\
\hline Sq3 & Firme & No es difícil. & $\begin{array}{l}\text { Una mezcla de agregados } \\
\text { porosos de } 2 \mathrm{~mm} \text { a } 10 \\
\mathrm{~cm} \text {; menos de } 30 \% \text { son } \\
<1 \mathrm{~cm} \text {. Algunos agrega- } \\
\text { dos (terrones) angulares, } \\
\text { no porosos pueden estar } \\
\text { presentes. }\end{array}$ & $\begin{array}{l}\text { Macroporos y } \\
\text { grietas presentes. } \\
\text { Alguna porosidad } \\
\text { dentro de los } \\
\text { agregados se } \\
\text { presenta como } \\
\text { poros o raíces. }\end{array}$ & $\begin{array}{l}\text { La mayoría de } \\
\text { las raíces están } \\
\text { alrededor de los } \\
\text { agregados. }\end{array}$ \\
\hline Sq4 & Compacta & Difícil. & $\begin{array}{l}\text { En su mayoría grandes > } \\
\text { de } 10 \mathrm{~cm} \text {, subangulares } \\
\text { no porosos; menos de } \\
30 \% \text { son menores de } 7 \\
\mathrm{~cm} \text {. Puede presentarse } \\
\text { estructura laminar. }\end{array}$ & $\begin{array}{l}\text { Pocos macropo- } \\
\text { ros y grietas. }\end{array}$ & $\begin{array}{l}\text { Todas las raíces } \\
\text { se concentran en } \\
\text { los macroporos y } \\
\text { alrededor de los } \\
\text { agregados. }\end{array}$ \\
\hline Sq5 & Muy compacta & Bastante difícil. & $\begin{array}{l}\text { En su mayoría grandes > } \\
\text { de } 10 \mathrm{~cm} \text {, muy pocos me- } \\
\text { nores de } 7 \mathrm{~cm} \text {, angulares } \\
\text { y no porosos. }\end{array}$ & $\begin{array}{l}\text { Muy baja, puede } \\
\text { haber macro- } \\
\text { poros. Puede } \\
\text { contener zonas } \\
\text { anaeróbicas. }\end{array}$ & $\begin{array}{l}\text { Pocas o ninguna, } \\
\text { si hay algunas se } \\
\text { concentran } \\
\text { en grietas. }\end{array}$ \\
\hline
\end{tabular}


Este método es rápido, de fácil uso y reproducibilidad, pero su interpretación es subjetiva y puede variar de un evaluador a otro; además, es sensible al contenido de agua del suelo al momento de realizar la prueba (Munkholm, 2011).

\section{Métodos semicuantitativos}

\subsection{Rompimiento del suelo por caída}

Este método permite determinar la friabilidad de un volumen -fragmento- de suelo y abordar principalmente la distribución del tamaño de fragmentos después de la caída e impacto de la masa de suelo sobre una superficie dura (Munkholm, 2011). La técnica consiste en obtener terrones de suelo y someterlos a caída libre sobre una superficie dura; los agregados o terrones pueden ser tomados directamente en campo u obtenidos a partir de bloques inalterados de suelo. Estos terrones o agregados se dejan caer desde una altura determinada, que de acuerdo con Hadas \& Wolf (1984), no debe exceder los $1.5 \mathrm{~m}$ (Figura 3), de tal forma que con el impacto, estos sufran un proceso de fragmentación a lo largo de sus planos de debilidad subdividiéndose en unidades naturales más pequeñas (Marshall \& Quirk, 1950). La distribución del tamaño de agregados resultante tras el impacto puede ser evaluada mediante la utilización de un juego de tamices, clasificando los tamaños de fragmentos de manera visual, o determinando gráficamente la distribución de tamaño de los mismos. Así mismo, se puede calcular el diámetro geométrico medio y el diámetro promedio ponderado de los agregados o fragmentos resultantes (Hadas \& Wolf, 1984; Munkhol et al., 2002b; Munkholm, 2011; Munkholm, et al., 2012). Este método es altamente sensible y dependiente del contenido de agua de la muestra de suelo por lo que se hace necesario determinar su contenido al momento de realización de la prueba para que los resultados sean comparables. El principio del método se fundamenta en la subdivisión de una masa de suelo en volúmenes más pequeños a partir de la energía liberada al momento del impacto sobre una superficie dura (Hadas \& Wolf, 1984).

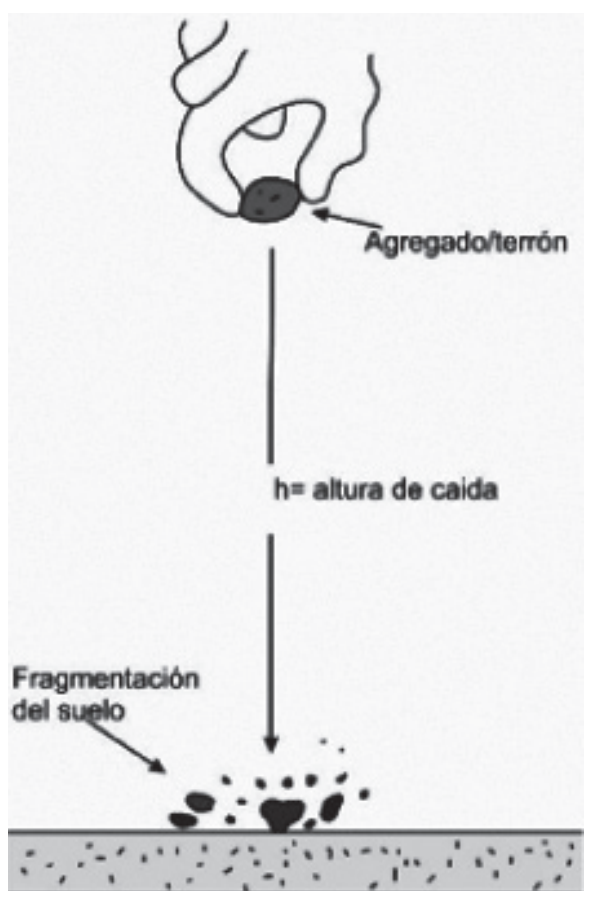

Figura 3. Esquema representativo del método de rompimiento del suelo por caída.

Este método es fácil de realizar y se puede llevar a cabo directamente en campo o en el laboratorio; de igual forma, requiere menos tiempo en su ejecución y equipos menos especializados con respecto a determinaciones cuantitativas, pero no ofrece información precisa sobre la resistencia del suelo (Munkholm, 2011).

\section{Métodos cuantitativos}

Existen métodos de determinación directa e indirecta de la friabilidad; entre los primeros, el método más utilizado es el de la resistencia del suelo al rompimiento -RR- (Utomo \& Dexter, 1981 y Dexter \& Watts, 2001). Entre los métodos de determinación indirecta se encuentran el de correlación con el índice de calidad estructural del suelo $-S$-, a partir de la curva de retención de agua del suelo (Dexter, 2004a), el de determinación a partir de la estimación de la porosidad estructural, y la estimación mediante funciones de pedotransferencia (Macks et al., 1996 y Dexter, 2004b). 


\subsection{Métodos de determinación directa \\ 3.1.1. Determinación de la resistencia del suelo al rompimiento (RR)}

La RR ha sido definida como la fuerza por unidad de área necesaria para provocar la ruptura en una muestra de suelo, es decir, para separarla (Dexter \& Watts, 2001). Esta característica es sensible a la microestructura por la función que cumplen las microfisuras en la resistencia del suelo y permite cuantificar la friabilidad (Dexter, 1988a; Hallett, 1995b; Watts \& Dexter, 1998). La resistencia del suelo depende del contenido de materia orgánica (Watts \& Dexter, 1998; Imhoff et al., 2002; Guimarães et al., 2009; Blanco-Moure et al., 2012), el contenido de agua (Munkholm \& Kay, 2002; Dexter, 2004b), la composición y concentración de la solución del suelo (Rahimi et al., 2000), la estabilidad de los agregados, los ciclos de humedecimiento y secado (Utomo \& Dexter, 1981), la cantidad y tipo de arcilla (Barzegar et al., 1994, 1995) y la cantidad de arcilla dispersable (Kay \& Dexter, 1992; Schjnning et al., 2011).

Según Munkholm (2011), la estimación de la RR es un procedimiento bien establecido y de uso común para cuantificar la friabilidad del suelo; adicionalmente, es un método cuyo enfoque es más objetivo con respecto a los métodos visuales descriptivos. La determinación de la friabilidad por este método se basa en que la resistencia del suelo a la fragmentación o desmoronamiento es controlada por la distribución de fisuras en el mismo (Watts \& Dexter, 1998). Estas fisuras corresponden a los espacios generados por los poros estructurales, los cuales bajo tensión mecánica, especialmente compresión, pueden alargarse y unirse con otros generando grietas de mayor dimensión que constituyen los límites de los nuevos fragmentos producidos por el desmoronamiento durante las operaciones de labranza (Dexter, 2004a).

Se conocen dos tipos de ensayos de resistencia del suelo al rompimiento: tensión y compresión; en el primero, la muestra de suelo es fragmentada en dos partes por efecto de la fuerza de tensión generada desde el centro de la muestra hacia sus extremos (Figura 4), por lo que al final, la muestra de suelo se fractura con una grieta que es perpendicular a la fuerza aplicada. Ensayos de resistencia a la tensión son complejos y difíciles de realizar, por lo que no son frecuentemente utilizados (Dexter \& Watts, 2001).

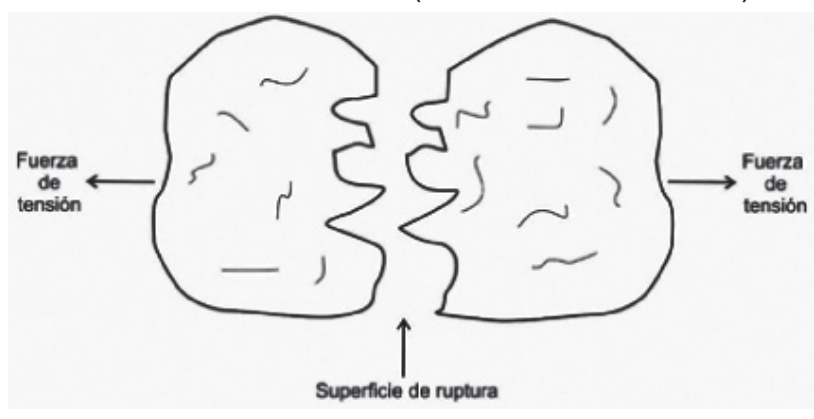

Figura 4. Esquema de agregado de suelo en la prueba de resistencia a la tensión.

Por el contrario, en el método de resistencia a la compresión la fuerza es aplicada a través del diámetro de la muestra tal como se observa en la Figura 5.

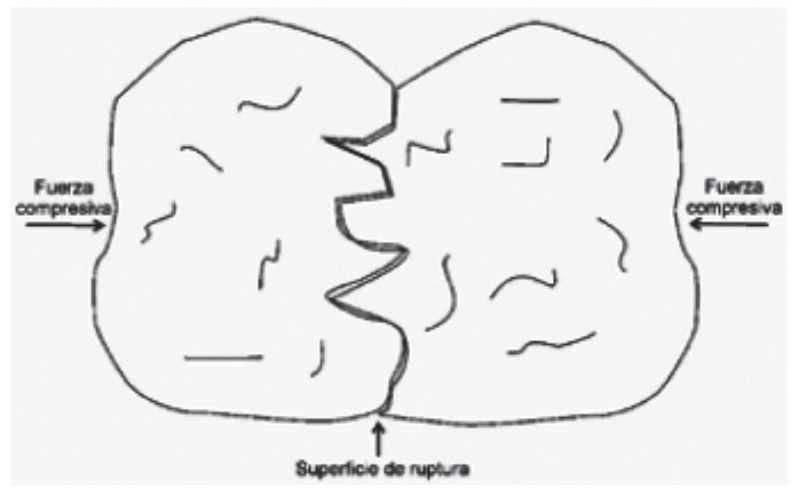

Figura 5. Esquema de agregado de suelo sometido a rompimiento en el ensayo de resistencia a la compresión.

Las muestras de suelo que se utilizan para la determinación de la RR corresponden a agregados o terrones de diferente tamaño, obtenidos de bloques indisturbados de suelo tomados en el horizonte y profundidad deseados; sin embargo algunos investigadores trabajan con agregados de suelo remoldeados (Barzegar et al., 1995). Los bloques son transportados al laboratorio evitando su rompimiento o debilitamiento y allí se realiza la separación de agregados por los planos de debilidad natural; dependiendo de las características del ensayo los agregados obtenidos pueden ser sometidos a humedecimiento a diferentes contenidos de agua, 
secado al aire, secado en horno a temperaturas bajas $\left(<60^{\circ} \mathrm{C}\right)$ o, incluso, secado en horno hasta los $105^{\circ} \mathrm{C}$. Los agregados secos se pasan a través de un juego de tamices que permite seleccionar el tamaño -o tamaños- de los agregados requeridos para la prueba. Algunos investigadores han empleado también muestras cilíndricas -sin disturbar-, para la determinación de la resistencia a la compresión del suelo (Dexter \& Kroesbergen, 1985; Munkholm et al., 2002a). El diámetro de los agregados debe ser conocido antes de hallar la resistencia a la compresión, sin embargo, el diámetro esférico efectivo de los mismos no se puede determinar con exactitud debido a su forma irregular. Dexter \& Kroesbergen (1985) describen cinco diferentes técnicas para la estimación del diámetro efectivo de los agregados:

Técnica 1: la muestra de suelo se pasa a través de un juego de dos tamices, cuyo valor de abertura de malla depende del rango de tamaño de agregado de interés. Los agregados colectados pasan a través del tamiz superior con una abertura de malla $d_{1}$ pero no través del tamiz inferior con abertura de malla $d_{2}$. El diámetro medio de los agregados $\left(D_{1}\right)$, cuyo valor es el asumido para todos los agregados individuales de la muestra, se determina a partir de la ecuación 1:

$$
\text { D1 }=\left(d_{1}+d_{2}\right) / 2
$$

Esta técnica que generalmente es utilizada para agregados de diferentes tamaños, ofrece como ventaja la posibilidad de trabajar con agregados de tamaño menor a $3 \mathrm{~mm}$ que normalmente ofrecen dificultad para su medición por otros métodos.

Técnica 2: los agregados una vez separados son medidos individualmente con calibrador $u$ otro dispositivo de medición adecuado. Dentro del rango de agregados seleccionado se debe medir el más grande (Dx), el intermedio (Dy) y el más pequeño: (Dz). En esta técnica el diámetro aritmético medio de los agregados $\left(D_{2}\right)$ es hallado a partir de la ecuación 2:

$$
D 2=(D x+D y+D z) / 3
$$

Este método presenta como ventaja que al determinar individualmente el diámetro de los agregados y la fuerza de compresión a la ruptura, permite reducir la varianza de los resultados de la RR.; sin embargo, no tiene buena aplicabilidad en agregados menores de $5 \mathrm{~mm}$ o en agregados de formas muy irregulares.

Técnica 3: los agregados individuales son medidos como en la técnica 2, pero el diámetro geométrico medio $\left(D_{3}\right)$ es calculado a partir de la ecuación 3:

$$
\mathrm{D} 3=(\mathrm{DxDyDz})^{1 / 3}
$$

Esta técnica presenta las mismas limitaciones que la número 2.

Técnica 4: se sigue el mismo procedimiento de tamizado de los agregados de la técnica 1 pero el diámetro efectivo es ajustado con base en las masas individuales de los agregados $\mathrm{M}$. El ajuste se realiza considerando que todos los agregados de la muestra tienen igual densidad aparente. Por lo tanto, si la masa media de un lote de agregados es $M_{0}$, se puede calcular la ecuación 4:

$\mathrm{D} 4=\mathrm{D} 1\left(\mathrm{M} / \mathrm{M}_{0}\right)^{1 / 3}$

donde D1 es el tamaño medio de los agregados seleccionados y $\mathrm{M}$ la masa de cada agregado. Este método es efectivo y confiable, ya que las masas individuales de los agregados se pueden obtener con rapidez y precisión mediante pesaje; así mismo, permite trabajar con agregados grandes y pequeños.

Técnica 5: en esta técnica, al igual que en la anterior, el diámetro individual de los agregados es ajustado de acuerdo con sus masas individuales (M), pero en esta la densidad media de los agregados (da) es conocida. Asumiendo que el diámetro de la esfera tiene el mismo volumen del agregado irregular, se tiene la ecuación 5 :

$$
\mathrm{D} 5=(6 \mathrm{M} / \pi \mathrm{da})^{1 / 3}
$$

Esta técnica ofrece una buena precisión en la medida de los agregados pero su desventaja es que solo se puede aplicar en suelos no expandibles o 
sometidos a ruptura en condición seca; adicionalmente, determinar la densidad aparente de cada agregado es muy dispendioso en tiempo y genera mayores costos.

Una vez determinada la masa individual y el diámetro efectivo, los agregados son sometidos individualmente a un esfuerzo de compresión, mediante la utilización de una prensa con dos platos paralelos para determinar la fuerza máxima necesaria para producir la ruptura del agregado (Figura 6) Dexter \& Kroesbergen (1985).

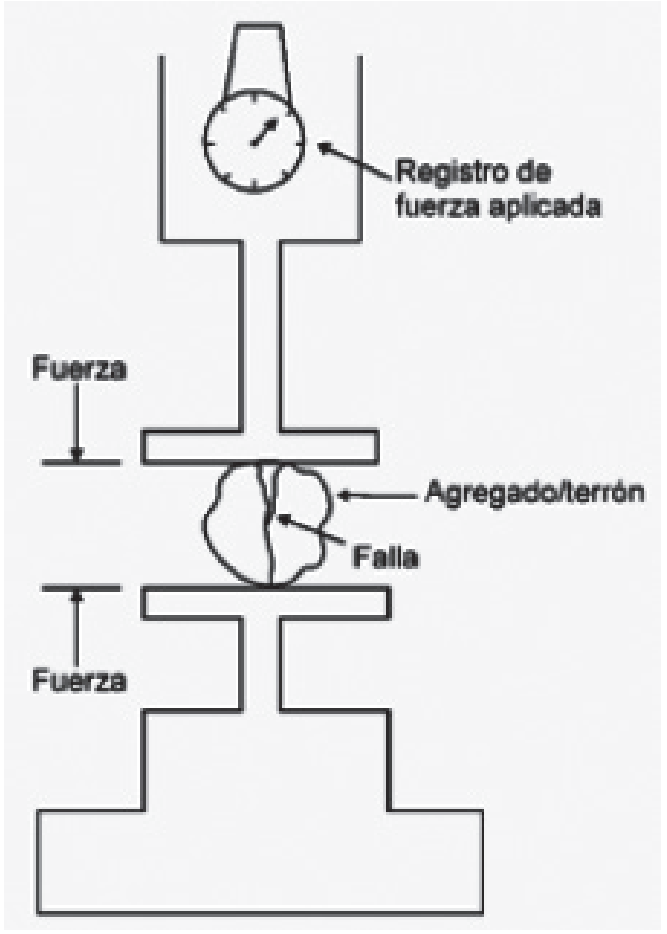

Figura 6. Determinación de la resistencia del suelo a la compresión.

La RR de los agregados se calcula mediante la ecuación (6), propuesta por Utomo \& Dexter, 1981; Dexter \& Kroesbergen, 1985; Dexter \& Watts, 2001:

$R R=0.576\left(P / D^{2}\right)$

En esta ecuación, RR -generalmente expresada en $\mathrm{kPa}$-, es la resistencia al rompimiento, $\mathrm{P}(\mathrm{N})$ el pico de fuerza registrado al momento de la ruptura del agregado, $\mathrm{D}(\mathrm{m})$ el diámetro efectivo de los agregados y el valor 0.576 corresponde a una constante de proporcionalidad (Dexter \& Kroesbergen, 1985,
Dexter \& Watts, 2001). Esta constante es la relación entre el esfuerzo de la compresión inconfinada polar y el esfuerzo de compresión producido dentro de la muestra; de esta constante se han propuesto valores que van desde 0.576 (Braunack et al., 1979) hasta 1.86 (Dexter, 1988b).

Basados en la RR, Watts y Dexter (1998) proponen el coeficiente de variación univariante como herramienta para estimar el índice de friabilidad, este coeficiente está dado por la ecuación 7:

$\mathrm{IF}=\frac{\sigma_{\mathrm{y}}}{\overline{\mathrm{Y}}} \pm \frac{\sigma_{\mathrm{y}}}{\overline{\mathrm{Y}} \sqrt{2 \mathrm{n}}}$

donde es el índice de friabilidad, es la desviación estándar de los valores medidos de RR y, es la media de la $R R$ medida en réplicas. El segundo término es el error estándar del coeficiente de variación.

Para interpretar la friabilidad del suelo a partir del coeficiente de variación de la RR se utiliza la clasificación presentada en la Tabla 4.

Tabla 4. Interpretación del índice de friabilidad estimado mediante el coeficiente de variación

\begin{tabular}{l|l}
\hline Índice de friabilidad & Calificación de la friabilidad \\
\hline$<0.1$ & No friable \\
\hline $0.1-0.2$ & Ligeramente friable \\
\hline $0.2-0.5$ & Friable \\
\hline $0.5-0.8$ & Muy friable \\
\hline$>0.8$ & Mecánicamente inestable \\
\hline
\end{tabular}

Fuente: Imhoff et al., 2002.

Los valores más altos de friabilidad en los terrones o agregados de mayor tamaño, en relación con los de tamaño menor, indica que los primeros tienen una RR baja, por lo que pueden ser más fácilmente fragmentados en sub-unidades más pequeñas de mayor $\mathrm{RR}$, con bajos requerimientos de energía para la labranza. Bajo esta condición el suelo requiere un mínimo de labranza para producir una buena cama de siembra, generando agregados de tamaño tal que ofrecen las condiciones óptimas para la germinación y el establecimiento de la plantas (Macks et al, 1996). 
Lo deseable en la capa arable del suelo es que los agregados pequeños tengan un menor índice de friabilidad (mayor RR), y en los grandes, este índice sea mayor (menor RR) (Utomo \& Dexter, 1981).

Algunos autores sugieren que la forma de los agregados influye también sobre la determinación de la RR. Seben et al. (2013) determinaron la RR tomando agregados en el rango de tamaño entre $12.5 \mathrm{y}$ $19 \mathrm{~mm}$, separándolos en tres tipos de formas y tratando de preservar su estructura inherente, así: (i) agregados de formas irregulares, (ii) agregados de formas esféricas y (iii) agregados de formas planas en la superficie de contacto con los platos de compresión. Estos autores encontraron que los agregados con formas irregulares presentaron resistencias a la compresión más altas $(306 \mathrm{kPa})$, comparados con los de formas esféricas (238 kPa) y los de formas planas en su superficie de contacto (129 kPa). Lo anterior se explica por los puntos de cada agregado que entran en contacto con los platos paralelos del equipo de compresión.

Sin embargo, la manipulación que se realiza de los agregados cuando se pulen y raspan de forma manual para adaptarlos a las formas referidas, puede debilitar los agregados y generar menores valores de RR, especialmente en los agregados más débiles y de menor tamaño.

\subsection{Métodos de determinación indirecta}

Se han propuesto algunos métodos que permiten inferir la friabilidad a partir del análisis de otras características del suelo, específicamente mediante la determinación de la curva de retención de agua del suelo (Dexter, 2004a) y la porosidad estructural del mismo (Guerif, 1990 y Munkholm et al., 2002b).

\subsubsection{Curva de retención de agua del suelo}

Este método se basa en la correlación entre el índice de calidad física del suelo $S$, establecido por Dexter (2004a), y la friabilidad. Para ello se utiliza el punto de inflexión de la curva de retención de agua del suelo (Figura 7), que corresponde al máximo valor del índice de calidad física del suelo $S$, como una medida de la condición óptima de friabilidad (Dexter, 2004b; Santos et al., 2011). De esta manera se tiene una medida indirecta de la friabilidad puesto que, por sí mismo, el método no permite proporcionar información sobre la resistencia o la fragmentación del suelo (Munkholm, 2011).

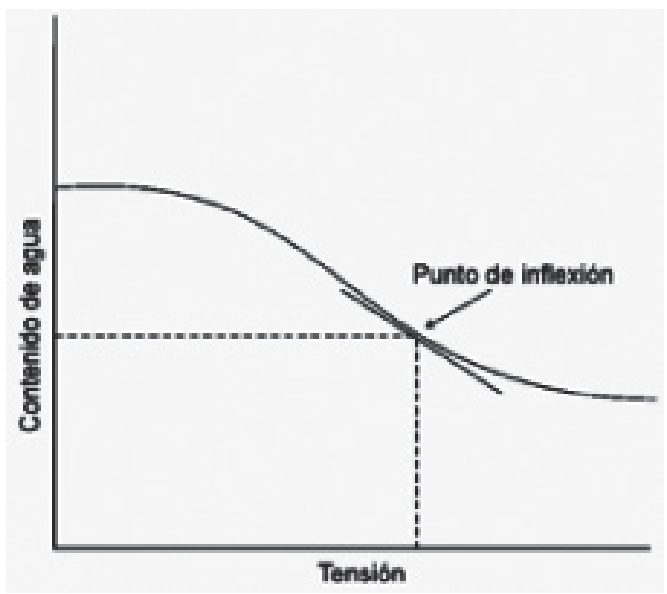

Figura 7. Punto de inflexión de la curva de retención de agua del suelo (máximo valor de S).

El índice de calidad física del suelo $S$, de acuerdo con Dexter (2004a), depende en gran medida de la porosidad microestructural del suelo, por lo tanto $S$ influye directamente en muchas de las principales propiedades físicas del suelo. Dexter \& Bird (2001), identificaron el contenido de agua que tiene el suelo en el punto de inflexión de la curva de retención de agua (máximo valor de $S$ ), como el contenido de agua óptimo para la labranza; en el que se produce el mayor número de pequeños agregados, o, por el contrario, el menor número de grandes agregados o terrones (Dexter, 2004b).

La correlación entre la friabilidad y la curva de retención de agua fue demostrada por Dexter (2004b), mediante su trabajo experimental con suelos de diferente friabilidad, contenido de agua y distribución de tamaño de agregados luego de ser labrados. Se demostró que la friabilidad fue lineal y correlacionó positivamente con el índice de calidad física, $S$.

\subsubsection{Porosidad estructural}

Este método consiste en estimar la friabilidad del suelo con base en la determinación del volumen de poros de diámetro equivalente mayor de $30 \mu \mathrm{m}$ (Munkholm, 2011). Guerif (1990) y Munkholm et al. (2002b) 
encontraron correlaciones significativas entre la resistencia del suelo y la macroporosidad y una correlación lineal negativa entre la resistencia del suelo y los volúmenes de poros mayores de 60 $\mu \mathrm{m}\left(R^{2}=0.54^{* * *}\right)$ y de poros mayores de $30 \mu \mathrm{m}$ $\left(R^{2}=0.35^{\star}\right)$. Para Munkholm $(2011,2012)$, el uso de la macroporosidad o porosidad estructural constituye una alternativa metodológica para evaluar la friabilidad; sin embargo, presenta como limitante que incluye tanto las grietas como los bioporos y, adicionalmente, ella en sí misma no proporciona información sobre la resistencia del suelo. Al igual que el método basado en la curva de retención de agua, descrito anteriormente, este método corresponde a una estimación indirecta de la friabilidad (Munkholm, 2011); por lo tanto, a partir de ellos no es posible determinar ni la RR, ni la fragmentación del suelo.

\subsubsection{Funciones de pedotransferencia}

La estimación de la friabilidad a partir de funciones de pedotransferencia se fundamenta en la medición de otras propiedades del suelo como la densidad aparente, sortividad, contenido de carbono orgánico e índice de estabilidad de los agregados y, a partir de modelos de regresión, realizados para un determinado número de muestras, obtener un estimativo de la friabilidad (Macks et al., 1996). Sin embargo, se requiere de mayor experimentación y una base de datos que incluya una gama amplia de tipos de suelos para confirmar la aplicabilidad de estas relaciones.

En general, son varias las opciones metodológicas disponibles para determinar la friabilidad de los suelos y, tal como señala Munkholm (2011), cada una de ellas mide diferentes aspectos de la friabilidad y se han utilizado para diferentes propósitos. Los métodos cualitativos y semicuantitativos son más accesibles a técnicos y agricultores, mientras que los métodos cuantitativos requieren un conocimiento y equipos más especializados.

\section{Conclusiones}

Existen diferentes métodos cualitativos para determinar la friabilidad del suelo, estos pueden realizarse directamente en campo de manera rápida y relativamente sencilla, pero su resultado es subjetivo y puede variar de un evaluador a otro. Adicionalmente se requiere determinar el contenido de agua del suelo al momento de realizar estas pruebas para obtener resultados aceptables, particularmente cuando se pretende determinar la friabilidad de diferentes tipos de suelos o en diferentes épocas de evaluación.

El método semicuantitativo -rompimiento del suelo por caída-, es sencillo, fácil de realizar y por lo tanto ofrece buen rendimiento en su ejecución, pero tiene la desventaja de proporcionar muy poca información sobre la resistencia del suelo y ser muy sensible a los cambios en el contenido de agua del suelo.

Los métodos cuantitativos ofrecen una información más objetiva, precisa y reproducible de la friabilidad, pero requieren para su realización equipos más complejos, preparación adecuada de la muestra, mayores lapsos de tiempo y personal más especializado.

Uno de los métodos más confiables para determinar la friabilidad de los suelos se fundamenta en la determinación de la resistencia del suelo al rompimiento. Este método cuantitativo ofrece la posibilidad de establecer la friabilidad del suelo mediante pruebas de compresión en terrones o agregados de diferente tamaño, en condición de suelo seco o a diferentes contenidos de agua.

\section{Literatura citada}

1. Ball, B.C., Batey, T., and Munkholm, L.J. (2007). Field assessment of soil structural quality - a development of the Peerlkamp test. Soil Use Manage. 23, 329-337.

2. Barzegar, A.R., Murray, R.X., Churchman, G.J.; Rengasamy, P. (1994). The strength of remoulded soils as affected by exchangeable cations and dispersible clay. Aust. J. Soil Res. 32, 185-199. 
3. Barzegar, A.R., Oades, J.M., Rengasamy, P., Murray, R.S. (1995). Tensile strength of dry, remoulded soils as affected by properties of the clay fraction. Geoderma 65, 93-108.

4. Blanco - Moure, N., Angurel, L.A., Moret-Fernández, D., López, M.V. (2012). Tensile strength and organic carbon of soil aggregates under long-term no tillage in semiarid Aragon (NE Spain). Geoderma 189-190, 423-430.

5. Braunack, M.V., Hewitt, J.S., and Dexter, A.R. (1979). Brittle fracture of soil aggregates and the compaction of aggregate beds. J. Soil Sci. 30, 653-667.

6. Dexter, A.R. (1988a). Advances in characterization of soil structure. Soil Till. Res. 11, 199-238.

7. Dexter, A.R. (1988b). Strength of soil aggregates and of aggregate beds. Catena Supplement 11, 35-52.

8. Dexter, A.R. (1997). Physical properties of tilled soil. Soil Till. Res. 43, 41-63.

9. Dexter, A.R. (2004a). Soil physical quality Part I. Theory, effects of soil texture, density, and organic matter, and effects on root growth. Geoderma 120, 201-214.

10. Dexter, A.R. (2004b). Soil physical quality: Part II. Friability, tillage, tilth and hard-setting. Geoderma 120, 215-225.

11. Dexter, A.R. \& Kroesbergen, B. (1985). Methodology for determination of tensile strength of soil aggregates. J. Agr. Eng. Res. 31, 139-147.

12. Dexter, A.R. \& Watts, C.W. (2001). Tensile strength and friability. In: Smith, K.A., Mullins, C.E. (Eds.), Soil and Environmental Analysis: Physical Methods. Segunda edición. Marcel Dekker Inc, New York, pp. 405-433.

13. Dexter, A.R. \& Bird, N.R. (2001). Methods for predicting the optimum and the range of soil water contents for tillage based on the water retention curve. Soil Till. Res. 57, 203-212.

14. FAO - Organization of the United Nations Food and Agriculture. (2009). Guide for soil description. Fourth edition. Rome, pp. 49-52.

15. Guérif, J. (1990). Factors influencing compactioninduced increases in soil strength. Soil Till. Res. 16, 167-178.

16. Guimarães, R.M.L., Tormena, C.A., Alves, S.J., Fidalski, J. \& Blainski, E. (2009). Tensile strength, friability and organic carbon in an oxisol under a crop-livestock system. Scientia Agricola. (Piracicaba, Brasil.), Vol.66, No4, 499-505.

17. Hadas, A. \& Wolf, D. (1984). Refinement and re-evaluation of the drop-shatter soil fragmentation method. Soil Till. Res. 4, 237-249.

18. Hallett, P.D., Dexter, A.R. \& Seville, J.P.K. (1995b). The application of fracture mechanics to crack propagation in dry soil. Eur. J. Soil Sci. 46, 591-599.

19. Imhoff, S., Da Silva, P.A. \& Dexter, A.R. (2002). Factors contributing to the tensile strength and friability of Oxisols. Soil Sci. Soc. Am. J. 66, 1656-1661.
20. Karlen, D.L., Erbach, D.C., Kaspar, T.C., Colvin, T.S., Berry, E.C. \& Timmons, D.R., (1990). Soil tilth: a review of past perceptions and future needs. Soil Sci.Soc. Am. J. 54, 153-161.

21. Kay, B.D. \& Dexter, A.R. (1992). The influence of dispersible clay and wetting/drying cycles on the tensile strength of a red-brown earth. Aust. J. Soil Res. 30, 297-310.

22. Macks, S.P., Murphy, B.W., Cresswell, H.P. \& Koen, T.B. (1996). Soil friability in relation to management history and suitability for direct drilling. Aust. J. Soil Res. 34, 343-360.

23. Marshall, T.J. \& Quirk, J.P. (1950). Stability of structural aggregates of dry soil. Aust. J. Agr. Econ. 1, 266-275.

24. Mullins, C.E., MacLeod, D.A., Northcote, K.H., Tisdall, J.M. \& Young, I.M. (1990). Hardsetting soils: Behaviour, occurrence and management. J. Adv. Soil Sci. 11, 37108.

25. Munkholm, L. J. (2011). Soil friability: A review of the concept, assessment and effects of soil properties and management (Review). Geoderma 167-168, 236-246.

26. Munkholm, L.J. \& Kay, B.D. (2002). Effect of water regime on aggregate-tensile strength, rupture energy, and friability. Soil Sci. Soc. Am. J. 66, 702-709.

27. Munkholm, L.J., Schjønning, P. \& Kay, B.D. (2002a). Tensile strength of soil cores in relations to aggregate strength, soil fragmentation and pore characteristics. Soil Till. Res. 64, 125-135.

28. Munkholm, L.J., Schjønning, P., Debosz, K., Jensen, H.E. \& Christensen, B.T. (2002b). Aggregate strength and mechanical behaviour of a sandy loam soil under long-term fertilization treatments. Eur. J. Soil Sci. 53, 129-137.

29. Munkholm, L.J., Heck, R.J. \& Deen, B. (2012). Soil pore characteristics assessed from X-ray micro-CT derived images and correlations to soil friability. Geoderma 181-182, 22-29.

30. Peerlkamp, P.K. (1959). A visual method of soil structure evaluation. Meded. v.d. Landbouwhogeschool en pzoekingsstations van de Staat te Gent. XXIV No. 24, pp. 216-221.

31. Rahimi, H., Pazira, E. \& Tajik, F. (2000). Effect of soil organic matter, electrical conductivity and sodium adsorption ratio on tensile strength of aggregates. Soil Till. Res. 54, 145-153.

32. Santos, G.G., da Silva, E., Marchao, R.B., da Silveira, P.M., Bruand, A., James, F. \& Becquer, T. (2011). Analysis of physical quality of soil using the water retention curve: Validity of the S-index. C. R. Geoscience 343, 295-301.

33. Schjønning, P., Elmhot, S., Munkholm, L. J. \& Debosz, K. (2002). Soil quality aspects of humid Sandy loams as influenced by organic and conventional long-term management. Agr. Ecosyst. Environ. 88, 195-214

34. Schjønning, P., De Jonge, L.W., Munkholm, L.J., Moldrup, P., Christensen, B.T. \& Olesen, J.E. (2011). Clay 
dispersibility and soil friability-testing the soil clay-tocarbon saturation concept. Soil Science Society of America Journal. Special section: Soil Architecture and Function. Vadose Zone J. doi:10.2136/vzj2011.0067.

35. Seben, G. de F., Corá, J. E., Fernández, C. \& Lal, R. (2013). Aggregate shape and tensile strength measurement. Technical Article. Soil Sci. 178 (6), 301-307.

36. Snyder, V.A., Vazquez, M.A., Martinez, G., Ramirez, L. \& Hadas, A. (1995). Controlled displacement technique for measuring soil friability. Soil Sci. Soc. Am. J. 59, 44-52.
37. USDA. (1993). Soil Survey Manual. Soil Survey Division Staff. Handbook No 18, pp. 173-181.

38. USDA. (2002). Field Book for Describing and Sampling Soils. Versión 2.0. National Soil Survey Center. Natural Resources Conservation Service, pp. 2-42/2-60.

39. Utomo, W.H. \& Dexter, A.R. (1981). Soil friability. J. Soil Sci. 32, 203-213.

40. Watts, C.W. \& Dexter, A.R. (1998). Soil friability: theory, measurement and the effects of management and organic carbon content. Eur. J. Soil Sci. 49, 73-84. 